\title{
PDK2: An Underappreciated Regulator of Liver Metabolism
}

\author{
Benjamin L. Woolbright ${ }^{1, *}$ and Robert A. Harris ${ }^{2}$ (D) \\ 1 Department of Urology, University of Kansas Medical Center, 3901 Rainbow Blvd, MS 1018, \\ Kansas City, KS 66160, USA \\ 2 Department of Biochemistry and Molecular Biology, University of Kansas Medical Center, \\ Kansas City, KS 66160, USA; raharris@iu.edu \\ * Correspondence: bwoolbright@kumc.edu; Tel.: +1-913-574-0517
}

check for

updates

Citation: Woolbright, B.L.; Harris, R.A. PDK2: An Underappreciated Regulator of Liver Metabolism. Livers 2021, 1, 82-97. https://doi.org/ 10.3390/livers1020008

Academic Editor: Vangelis Kondylis

Received: 22 March 2021

Accepted: 5 May 2021

Published: 11 May 2021

Publisher's Note: MDPI stays neutral with regard to jurisdictional claims in published maps and institutional affiliations.

Copyright: (c) 2021 by the authors. Licensee MDPI, Basel, Switzerland. This article is an open access article distributed under the terms and conditions of the Creative Commons Attribution (CC BY) license (https:// creativecommons.org/licenses/by/ $4.0 /)$.

\begin{abstract}
Pyruvate metabolism is critical for all mammalian cells. The pyruvate dehydrogenase complex couples the pyruvate formed as the primary product of glycolysis to the formation of acetyl-CoA required as the primary substrate of the citric acid cycle. Dysregulation of this coupling contributes to alterations in metabolic flexibility in obesity, diabetes, cancer, and more. The pyruvate dehydrogenase kinase family of isozymes phosphorylate and inactive the pyruvate dehydrogenase complex in the mitochondria. This function makes them critical mediators of mitochondrial metabolism and drug targets in a number of disease states. The liver expresses multiple PDKs, predominantly PDK1 and PDK2 in the fed state and PDK1, PDK2, and PDK4 in the starved and diabetic states. PDK4 undergoes substantial transcriptional regulation in response to a diverse array of stimuli in most tissues. PDK2 has received less attention than PDK4 potentially due to the dramatic changes in transcriptional gene regulation. However, PDK2 is more responsive than the other PDKs to feedforward and feedback regulation by substrates and products of the pyruvate dehydrogenase complex. Although underappreciated, this makes PDK2 particularly important for the minute-to-minute fine control of the pyruvate dehydrogenase complex and a major contributor to metabolic flexibility. The purpose of this review is to characterize the underappreciated role of PDK2 in liver metabolism. We will focus on known biological actions and physiological roles as well as what roles PDK2 may play in disease states. We will also define current inhibitors and address their potential as therapeutic agents in the future.
\end{abstract}

Keywords: pyruvate dehydrogenase kinase; pyruvate dehydrogenase; metabolism; PDK2; cancer; inflammation; diabetes

\section{Introduction}

The pyruvate dehydrogenase kinase (PDK) family of isozymes consists of four isozymes whose primary known function is to phosphorylate and inactivate pyruvate dehydrogenase (PDH), preventing the conversion of pyruvate to acetyl-CoA [1]. This leads to a shift in metabolism away from the citric acid cycle and towards alternate metabolism of pyruvate. This has far-reaching effects on metabolism and is a major point of regulatory control in the body's ability to shift fuel selection. Classically, one of the most well-known outcomes of PDH phosphorylation is enhanced conversion of glucose to lactate and an increase in ATP production by glycolysis relative to oxidative metabolism. This is commonly referred to as aerobic glycolysis because it occurs in the presence of oxygen that could support complete oxidation of glucose to $\mathrm{CO}_{2}$ and $\mathrm{H}_{2} \mathrm{O}$. Aerobic glycolysis occurs in numerous cell types, but is most well studied in cancer [2,3]. Increasingly though, research indicates that PDK-mediated phosphorylation of PDH yields an array of metabolic changes designed to support anabolism during times of cellular stress and proliferation [4,5]. PDKs are not only critical for normal maintenance of metabolism, but are also critical regulators of cellular homeostasis in a wide range of pathologies. Targeting PDKs therapeutically is being investigated in multiple disease states. 
The four PDK isozymes are differentially expressed throughout the body [1]. As their position on the genome also varies widely, we surmise that this may represent evolutionary differences as individual tissue niches developed the capacity to regulate $\mathrm{PDH}$ independently. Recent data indicate that most tissues are dominated enzymatically by a single isozyme, confirming the hypothesis that there is widespread tissue-specific regulation of PDKs [6]. A notable exception to the idea of a single PDK being primarily responsible for most PDK activity is the liver [6]. The liver constitutively expresses high levels of PDK1 and PDK2 and both likely contribute significantly to overall hepatic PDH activity [6]. This is not overtly surprising because of the diverse metabolic capacities of the liver and its need to be able to adapt. The liver also expresses lower quantities of PDK4 natively; however, PDK4 is highly inducible and repressible by a number of different stimuli $[7,8]$. Because of the highly inducible nature of PDK4, it has been extensively examined in the liver as it is thought to function largely as a metabolic regulator during times of cellular stress or during nutrient deprivation [4,5]. These drastic changes in expression, in concert with functional studies demonstrating a role for PDK4 in metabolic adaptation to diverse stimuli, have led researchers to extensively study PDK4 in multiple tissues, including the liver [9-12]. In contrast, PDK2 has been less well studied, potentially due to the fact that while its enzymatic activity is tantamount to liver function, it generally does not undergo the same degrees of genetic regulation and its established functional roles in diseases such as diabetes are relatively minor compared to PDK4. In spite of this, recent studies with PDK2 $2^{-/-}$mice and with inhibitors with preferential specificity for PDK2 or PDK2/4 all indicate that constitutive PDK2 expression likely plays a role in many pathogenic processes, similar to PDK4. More importantly, combined knockdown or inhibition of PDK2/PDK4 provides more robust results than knockdown of either component alone, yielding a rationale for advanced drug targeting of specific PDKs in specific disease states. The purpose of this review is to understand how PDK2 activity plays a role in a diverse array of liver diseases. We will describe the function and role of PDK2, define transcriptional and allosteric regulators, address putative inhibitors and identify diseases where PDK2 plays an active functional role.

\section{The PDKs and PDH}

The PDH complex is a large multi-meric enzymatic complex that carries out three separate reactions to convert pyruvate to acetyl-CoA [4]. The components, labeled as E1 (pyruvate dehydrogenase), E2 (dihydrolipol transacetylase), E3BP (E3 binding protein), and E3 (dihydrolipoyl dehydrogenase), represent three different enzymatic complexes which act in succession to generate acetyl-CoA from pyruvate. The PDKs phosphorylate and inactivate the PDH complex through phosphorylation of the E1 subunit of PDH at three serine sites, commonly referred to as site 1, 2 and 3 [4]. This is counter-regulated enzymatically by two pyruvate dehydrogenase phosphatase enzymes (PDP1/PDP2) that dephosphorylate and re-activate phosphorylated PDH [13]. The effects of PDKs, including PDK2, are widely attributed to their ability to phosphorylate PDH. This topic has been reviewed extensively and many high quality resources are available for in-depth mechanistic understanding of the interactions between the PDKs and PDH [4,5,14]. While it is possible that one or more of the PDKs have other substrates that they can phosphorylate [15], by far the most commonly proposed mechanism is phosphorylation of PDH with subsequent changes in metabolism.

\section{Physiological Roles of PDKs}

\subsection{PDKs in Normal Physiology}

The primary roles of the PDKs is to constrain PDH activity and to divert energy metabolism to alternative means when necessary through control of PDH [16]. The liver $\mathrm{PDH}$ is less active (more phosphorylated) in the fasted state than in the well-fed state; however, even in the very well-fed state, PDH is not fully dephosphorylated and not fully active. In other words, a considerable reserve of PDH activity exists in the liver even in 
the well-fed state. The activity state of PDH is not static and changes rapidly depending upon the availability of pyruvate and the need for acetyl-CoA. Maximum activation of the pyruvate dehydrogenase by complete dephosphorylation would rapidly deplete the liver of citric acid cycle intermediates and pyruvate and thus tight but flexible control of PDH activity is required for normal tissue maintenance. Suppression of pyruvate dehydrogenase activity by PDKs, even in the fed state, conserves pyruvate for synthesis of oxaloacetate by pyruvate carboxylase (anaplerosis) and can thus fuel the citric acid cycle indirectly in the event of a sudden drop in glucose/pyruvate availability [4].

During extended periods of minimal glucose availability or fasting, glucose levels are reduced, and thus the primary fuel for many tissues, glucose, must come from other sources. As glucose levels decrease, the PDH complex is rapidly inactivated in multiple tissues via phosphorylation by PDKs to conserve pyruvate and other compounds readily converted to pyruvate, namely lactate (via lactate dehydrogenase) and alanine (via alanine aminotransferase) [4]. This is due to upregulation of PDKs in target tissues by serum free fatty acids that are liberated from fat stores and reductions in serum insulin levels that potently regulates PDK activity [9]. In addition to conservation of pyruvate for anaplerosis, inhibition of PDH by PDKs increases the amount of three-carbon compounds available (pyruvate/lactate, alanine) which partially fuels the continuous need for glucose through gluconeogenesis. Thus, PDK activity is essential to life's adaptation to nutrient deprivation. This may explain why multiple PDK isozymes are present in many organisms, as evolutionary pressure for organisms who cannot survive periods of nutrient scarcity would likely be high. As such, PDKs in normal physiology serve as a brake on uncontrolled mitochondrial oxidation of pyruvate in order to conserve three-carbon intermediates for alternative energy production, fuel anaplerotic reactions such as those carried out by pyruvate carboxylase, and provide stable sources of glucose during periods of low nutrient availability.

\subsection{Specific Roles for PDK2 in Normal Physiology:}

PDK2 appears to be the PDK most capable of rapid regulation of PDH activity under physiological conditions in the liver. Of the four PDKs, PDK2 is best suited to oppose the activation of the pyruvate dehydrogenase by the PDPs. This is because PDK2 is constitutively expressed, has greater activity than the other PDKs for phosphorylation of site 1, the most important regulatory site, is more sensitive to inhibition by pyruvate than the other PDKs, and is more sensitive to activation by NADH and acetyl-CoA via the reduced and acetylated-lipoyl groups on the E2 subunit of the PDH complex $[4,17,18]$. The liver has to respond rapidly to changes in the availability of glucose provided by the diet as well as changes in other nutrients such as lactate, alanine, and pyruvate delivered from the peripheral tissues by the blood. The constitutive expression and the kinetic characteristics of PDK2 make it critically important for metabolic flexibility.

The presence of multiple PDKs in many tissues, including liver, has made understanding the role of each individual PDK more difficult. PDK2 is constitutively expressed at high levels and controls a considerable degree of basal PDH activity in the liver $[6,8,19]$. PDK1 is also functionally expressed in liver and exerts some control over PDH phosphorylation [6]. PDK1 is regulated by a number of factors, including hypoxia-inducible factor- $1 \alpha$ (HIF-1 $\alpha$ ) [20]. PDK2 is likely also regulated by HIF-1 $\alpha$, but possibly to different degrees in different tissues, and PDK2 and PDK1 are known to co-regulate PDH activity in animal models of genetic loss [21,22]. Knockout of PDK2 reduces overall hepatic PDK activity by approximately $40 \%$ [6]. It was hypothesized that this reduction is compensated heavily by increases in PDK1 activity in the liver during loss of PDK2; however, this could not be confirmed in PDK1 $1^{-/}$animals as PDK1 ${ }^{-/}$animals also have dramatic reductions in PDK2 expression [6]. The authors hypothesized that this may be due to stabilization of PDK2 by PDK1, although this has not been directly tested [6]. While these data may argue in part for a co-dominant role for PDK1, understanding this effect will require a better delineation of PDK1 and PDK2. Given their similarity in activity and that the common 
losses seen in one protein when the other is knocked down or otherwise depleted, it is possible that PDK1 and PDK2 form heterodimers that are more stable than the corresponding homodimers. While this may make understanding their effects challenging, experiments directly demonstrating this would be highly useful to the field.

In spite of the obvious effects of PDK2 on physiologic PDH activity, delineating clear effects of PDK2 in vivo has been difficult. For example, PDK2 is only minimally involved in regulating blood glucose during fasting. In contrast to PDK4 ${ }^{-/-}$animals, $\mathrm{PDK}^{-/-}$animals have no obvious phenotype and their response to fasting is similar to WT animals with no change in blood glucose [12]. This is likely due to heavy compensation by PDK1 $[6,22]$ and upregulation of PDK4 during periods of nutrient deprivation in multiple tissues $[8,9,19]$. These data support a hypothesis where PDK4, as the highly inducible form of PDK, primarily regulates $\mathrm{PDH}$ during periods of stress and is responsible for the liver's capacity to adapt to external changes to alternate fuel selection, whereas PDK2 serves as a rapid tuning agent for fuel selection during both homeostasis and during periods of cellular stress or pathology, potentially in concert with PDK1.

\section{Transcriptional Regulators of PDK2 Expression}

While PDK4 levels are inducible to much higher levels than PDK2, both enzymes are transcriptionally regulated by a number of factors in addition to regulation by substrates and products of $\mathrm{PDH}$ which generate tight control over $\mathrm{PDH}$ phosphorylation. Primarily, starvation or a "fasted state" is an inducer of PDK activity including PDK2 and, to a larger degree, PDK4 [12,23]. This is an important physiological mechanism as upregulation of PDK2 (and PDK4) during starvation reduces conversion of pyruvate to acetyl-CoA during periods of starvation and conserves pyruvate for gluconeogenesis, glycogen storage, and anaplerotic reactions as previously mentioned $[8,19]$. While PDK4 is potently regulated by factors such as insulin, thyroid hormone, peroxisome proliferator-activated receptor proteins (PPARs), and more, PDK2 is less extensively transcriptionally regulated [7,24]. Even still, we will briefly discuss signaling pathways which regulate PDK2 and the implications thereof.

Insulin regulates PDK2 expression although not to the same degree as it does PDK4. Importantly, it should be noted that despite the relatively lower degree of regulation, physiological PDK2 expression often remains elevated above PDK4 levels, even during starvation/insulinemia [25]. Reduced insulin levels consistent with diabetes and starvation modestly upregulate PDK2 in the liver [12]. In the heart, these effects are reversible through either re-feeding or administration of insulin, confirming a mechanistic role of insulin [26]. These data are supported by studies in the Pima Native American population demonstrating reductions in PDK2 when insulin is given to non-insulin-dependent diabetes mellitus patients [27]. The increase in PDKs associated with diabetes or starvation is likely mediated through forkhead box protein O1 (FOXO1) $[28,29]$. Insulin is a potent negative regulator of FOXO1 through phosphorylation events catalyzed by protein kinase $\mathrm{B}$ [30]. FOXO1 is a transcription factor activated during starvation broadly regulates gene expression, including upregulation of other genes involved in glycolysis [31]. Regulation of PDKs by insulin and FOXO1 thus complete a signaling loop that allows for detection of nutrient status and a subsequent response. Because this pathway is dysregulated in patients with insulinemia or diabetes, inhibition of PDKs has been proposed as a means for treating diabetes (see section PDK2 and the metabolic syndrome). Other potential regulators include the peroxisome proliferator-activated receptors and their ligands although the degree to which they regulate PDK2 may be tissue dependent [32].

A diverse array of transcription factors regulate PDK4, but many of these did not extend to PDK2 [7,24]. A notable exception is RORalpha, as genetic overexpression of ROR $\alpha$ downregulates PDK2 through p21 [33]. This was somewhat surprisingly found not to occur through p53, despite the fact that p53 is a major regulator of p21, and regulates PDK2 in carcinoid cancer [34]. Little effect was found on PDK1, PDK3 or PDK4 and thus ROR $\alpha$ may be a PDK2-specific regulator. Conditions that damage DNA, such as chronic 
inflammation in obesity and aging, may activate p53, which indirectly increases PDH activity by downregulating expression of PDK2.

More information is needed to understand how PDK2 is regulated. A brief figure summarizing transcriptional regulation of PDK2 is presented here (Figure 1). While we have hypothesized currently that levels of PDK2 are less overtly regulated at the transcriptional level, it is also possible that we do not yet understand what molecules transcriptionally regulate PDK2 efficiently, i.e., $\operatorname{ROR} \alpha, \mathrm{p} 21$, and $\mathrm{p} 53$. As we have noted previously, PDK2 is far more responsive to allosteric regulators such as ADP, acetyl-CoA, and pyruvate than it is to transcriptional control [35]. Minimal transcriptional control of PDK2 emphasizes the idea that PDK2 is primarily regulated by both the substrates and products of the PDH complex. Further understanding how PDK2 is regulated will likely benefit future studies in many diseases. This is discussed in greater detail in later sections.

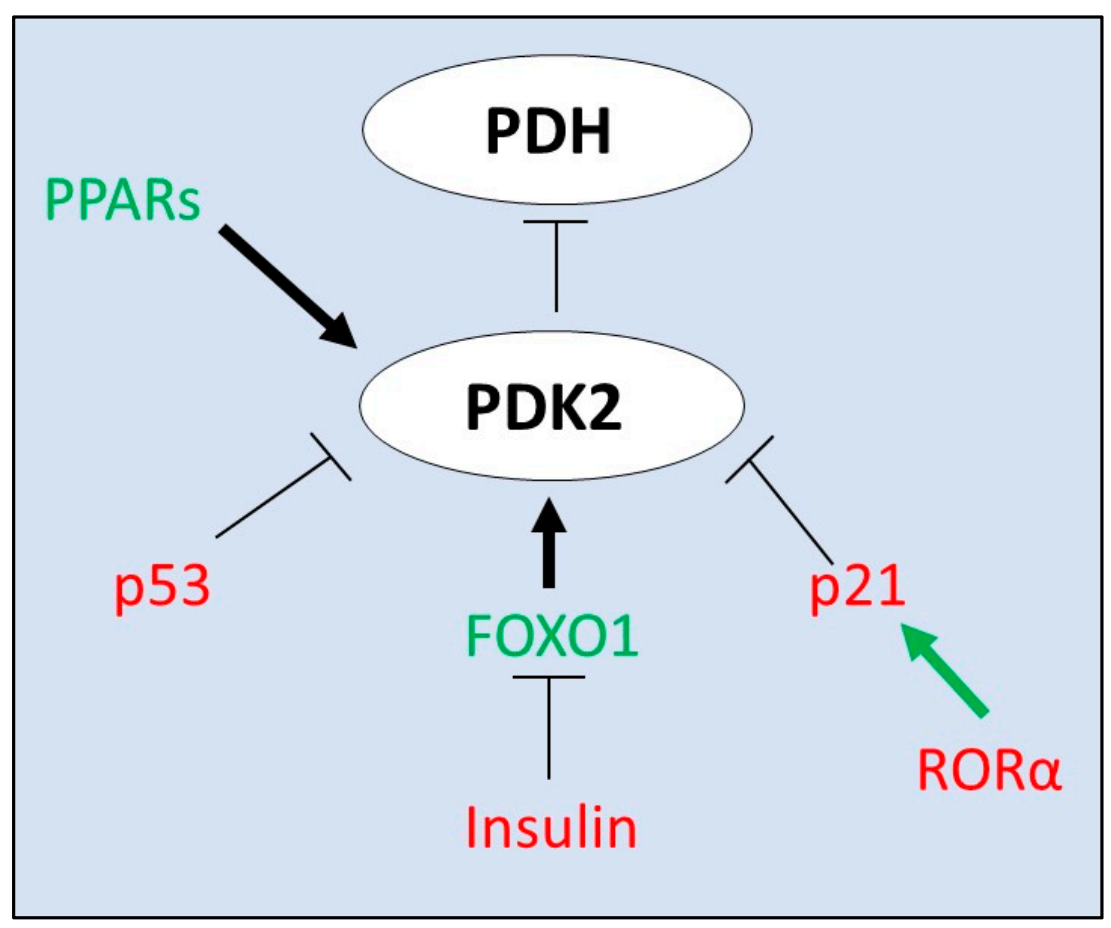

Figure 1. Transcriptional regulation of PDK2. PDK2 is primarily regulated by allosteric regulators. Known transcriptional regulators include proteins such as p21 and p53. Per Figure 1, the majority of regulation of PDK2 is through regulation by its co-factors and other substrates/products. PPARsperoxisome proliferator-activated receptor, PDK—-pyruvate dehydrogenase kinase, PDH-pyruvate dehydrogenase, ROR $\alpha$-RAR-related orphan receptor alpha, and FOXO1—forkhead box protein O1.

\section{PDK2 Inhibitors}

A number of PDK2 inhibitors have been identified (Table 1). Unfortunately, because of substantial overlap between the PDKs, as well as similarity to other kinases, some PDK2 inhibitors also have activity against other proteins [17]. We have tried to identify the most relevant inhibitors based on their binding site, potency and potential as prototypical therapeutic agents. 
Table 1. Prototypical PDK2 inhibitors. PDK2 inhibitors listed by efficacy, and target site of action.

\begin{tabular}{|c|c|c|c|c|}
\hline Inhibitor & Target & Efficacy & Notes & Refs \\
\hline Dichloroacetate & pyruvate binding site & millimolar & common inhibitor & [4] \\
\hline PS10 & ATP binding site & micromolar & $\begin{array}{l}\text { high specificity for PDKs } 2 \text { and } 4 \text {, and to a } \\
\text { lesser degree PDK1 }\end{array}$ & [36] \\
\hline azd7545 & PDH E2 lipoyl domain & micromolar & higher specificity for PDK1 and PDK2 & {$[37,38]$} \\
\hline 2-chloropropionate & pyruvate binding site & millimolar & DCA analog & [4] \\
\hline radicicol & ATP binding site & micromolar & also inhibits HSP90 & [39] \\
\hline VER-246608 & ATP binding site & millimolar & pan-isoform inhibitor & [40] \\
\hline $\begin{array}{l}\text { Compound 17, PS10 } \\
\text { derivative }\end{array}$ & $\begin{array}{l}\text { ATP binding site } \\
\text { inhibitor }\end{array}$ & micromolar & poorly defined thus far & {$[36,41]$} \\
\hline
\end{tabular}

\subsection{Dichloroacetate}

The pyruvate binding site is located at the N-terminal site of PDK2 and serves as a molecular sensor for pyruvate, a major allosteric regulator of PDKs [42,43]. As pyruvate is the primary substrate metabolized by the $\mathrm{PDH}$ complex, negative allosteric regulation by pyruvate serves to enhance the production of acetyl-CoA through inhibition of PDK isozymes [35,42]. In simpler terms, when intracellular pyruvate levels are high, PDKs are typically inhibited and the PDH complex becomes more active. Pyruvate and other endogenous physiological inhibitors such as $\mathrm{NAD}^{+}$and $\mathrm{ADP}$ all serve similar roles in this regard [44]. PDK2 is highly sensitive to these mechanisms, displaying synergistic inhibition by pyruvate and other endogenous inhibitors such as ADP $[35,42]$.

Dichloroacetate (DCA) is a pan-PDK inhibitor that affects PDKs 1-4; however, it inhibits PDK2 more potently than the other PDKs [1,4]. DCA binds to the N-terminal putative pyruvate binding site and allosterically inhibits the action of all PDKs, including PDK2 [17,45]. All PDKs contain an "ATP-lid" like structure that provides access to the nucleotide binding region. Specific amino acid residues (R250, T302 and Y320) mediate the interaction between DCA and PDK2 by allowing the open and closed confirmations of the ATP-lid that are associated with either activity or inactivity [18]. This is accomplished by allosteric interactions that alter the shape of the active site and prevent PDKs from phosphorylating PDH E1 $\alpha[4,17,18,45]$. The effects of DCA are partially mediated by interactions between the $\mathrm{N}$-terminal and $\mathrm{C}$-terminal ends of the protein, as mutations in Aspartate-382 and Tryptophan-383 increase DCA IC50s substantially due to altered binding with N-terminal partners Tyrosine-145 and Arginine-149 [17,45]. DCA inhibits PDK2 with an IC50 of $\sim 290 \mu \mathrm{M}$ and is a prototypical inhibitor of PDK2 [4].

DCA is a chlorinated analogue of acetic acid that does not demonstrate particularly strong drug-like characteristics [1]. Because of its small size and weak affinity for PDKs as well as the DCA binding site being "buried" or largely inaccessible to inhibitors, DCA typically requires millimolar concentrations to initiate an effect in vitro $[1,4,46]$. This may also be in part due to the need for active uptake by the sodium transporter SLC5A8 as well as degradation of the compound through metabolism by glutathione transferase zeta intracellularly [47-49]. These data are supported by studies in isolated mitochondria and perfused rat hearts, which demonstrate intact cells require far higher concentrations for inhibition of PDKs than isolated mitochondria [46]. This is largely due to lack of cellular uptake, a problem removed in isolated mitochondria, but may also be in part due to DCA concentrating in the mitochondrial matrix when it does enter the cell [46]. Regardless, DCA is rapidly absorbed in vivo and widely distributed to various tissues, particularly upon repeat dosing due to inhibition of glutathione S-transferase zeta [50]. Prior clinical trials indicate that effective clinical doses of $10 \mathrm{mg} / \mathrm{kg}$ and above result in reduction in serum lactate in less than an hour and is consistent with data demonstrating that the PDH complex is widely stimulated within $15 \mathrm{~min}$ in patients [50,51]. As such, although DCA is not what would classically be considered "drug-like", its actions in vivo are established. Unfortunately, DCA pharmacokinetics in vivo present challenges in using DCA as a drug. Patient genotype plays very directly into its utility as a drug as patients with increased 
glutathione transferase activity metabolize the drug dramatically more rapidly which alters bioavailability [52]. Moreover, the use of DCA as a therapeutic is further complicated by reversible peripheral neuropathy that occurs near doses that are effective in vivo $[53,54]$. DCA has been used clinically in a number of disease states through early phase clinical trials; however, due to either modest toxicity or lack of potency, it is not currently indicated for any specific disease.

In laboratory models, DCA has routinely been used to validate findings demonstrated after genetic knockdown or knockout of PDK2. It should be noted that while DCA is an effective inhibitor of PDK2, its effect is not limited to PDK2. In contrast, DCA blocks the action of all PDKs and thus is a highly potent stimulator of the PDH complex regardless of its actions on PDK2. Furthermore, the degree to which some of DCA's effects on diseases such as cancer are sometimes poorly linked to its metabolic effects and may be more complex $[55,56]$. Moreover, conflicting data on the role of PDK:PDH have led to confusion about mechanisms and potential usefulness of PDK inhibitors in cancer. Loss of PDH, the presumed target of the PDKs, has been reported to reduce cancer growth in prostate cancer [57]. Anchoring these data, an inhibitor of PDH, CPI-613 reduces cancer growth in a number of different cellular models [58]. This is surprising as the assumption would be that loss of PDH, the target of PDKs, would have the opposite effect. A potential explanation for these findings is that metabolic disruption generally adversely affects cancer. It has been proposed that cancer cells require not only high rates of precursor material but also the high capacity for the generation of precursor material, i.e., high rates of metabolic flux [59]. It is possible that disturbing either PDK or PDH alters metabolic flux rates in critical pathways despite the seemingly paradoxical nature of the findings [59]. Regardless, DCA is the prototypical inhibitor used in a variety of models and has commonly been demonstrated to impact disease states or synergize with other treatments to further their effects.

\subsection{ATP Binding Site/PS10}

The ATP binding site represents a ubiquitously targeted site for inhibition of kinases, including PDK2. Because of this, many inhibitors may have overlapping activity as a PDK inhibitor due to similarity with the ATP binding site of other proteins. Furthermore, the PDK superfamily of isozymes belongs to the GHKL (gyrase B, HSP90, Hisitidine kinase-like mutL) family of enzymes [36,60]. As such, many inhibitors of the PDK family of isozymes also have known activity against HSP90, and likely other kinases, and vice versa $[17,40]$. Shared motifs between these members include the ATP binding site and the "ATP lid" structure that participates in protein-protein interactions, and allows access to the nucleotide binding pocket [36,60]. Similarities between the PDK family and HSP90 family are potentially best exemplified by evidence that change in a single functional group of an HSP90 inhibitor results in a dramatic switch from an HSP90 inhibitor to a PDK inhibitor, with relatively high specificity [41]. Dual PDK/HSP90 inhibitors have also been generated both incidentally (radicicol) and intentionally for the treatment of cancer [37]. The similarities between HSP90 and PDKs complicates drug development as the diverse affects found by heat shock inhibition complicate mechanistic understanding of experiments and may have detrimental effects in some disease states. Moreover, HSP90 inhibition can cause an undesirable heat-shock response that paradoxically alters cellular signaling and interferes with disease progression in diseases such as cancer.

Some recent work at developing inhibitors with improved potency have focused on the ATP binding site. ATP competitive inhibitors such as VER-246608 have demonstrated anti-cancer activity and inhibit PDH phosphorylation [38]. The compound PS10 has improved potency (PDK2 IC50 $<1 \mu \mathrm{M}$ ), improved pharmacokinetics as compared to DCA (T1/2 3 h vs. $1 \mathrm{~h}$ ), and as of yet few known major side effects [41,50]. In contrast to the allosteric inhibition generated by DCA, PS10 directly binds to and inhibits the ATP binding site [41]. Kinome inhibition analysis indicates that PS10 has much higher affinity for PDKs (particularly 2 and 4) versus other kinases with minimal effect at HSP90 [37]. Further medicinal chemistry efforts have generated an improved candidate for inhibition 
of liver PDK2 [61]. Compound 17 (lead compound) is cleared from plasma faster than PS10; however, it rapidly distributes to liver where it is cleared slowly, resulting in better pharmacokinetics for inhibition of PDK2 (and PDK4) in the liver [61]. Studies in models of diet-induced obesity indicate that PS10 reduces hepatic steatosis, reduces blood glucose levels after glucose challenge and reduces serum lactate levels indicative of activation of the PDH complex [41]. Similar results were found when animals were treated with Compound 17 instead of PS10 [61]. These compounds remain under development; however, the initial findings indicate that these compounds are more likely to specifically inhibit PDK2 than DCA and may have therapeutic usage in diabetes, cancer, and more.

\subsection{Lipoyl-Binding Domain/AZD7545}

PDKs bind the L2 lipoyl-binding domain of PDH E2 and subsequently phosphorylate and inactivate PDH E1 $\alpha$. Inhibitors of binding to the L2 domain of PDH E2 serve as inhibitors of PDKs by preventing binding with the PDH complex. Experimentally, these inhibitors typically demonstrate substantial shifts in IC50 values when the E2:E3BP core is added in addition to PDH E1 in order demonstrate their effect, which further solidifies the dependence on E2:E3BP [17,62]. AZD7545 is one of the most potent PDK1 and PDK2 inhibitors currently known, but is dependent on inhibition at the PDH E2 domain. AZD7545 dramatically inhibits PDK2 activity when the E2 complex is added to assays assessing PDK/PDH activity, an effect which is lost when E2 is not added indicating an E2-specific effect [17]. AZD7545 inhibits PDK1 and PDK2 activity at similar levels ( 36 and $6 \mathrm{nM}$, respectively) by directly inserting in the lipoyl-binding pocket that binds the lipoylbinding domains in PDH E2 [62,63]. In isolated rat hepatocytes, AZD7545 increases PDH phosphorylation at a concentration of $5 \mathrm{nM}$ [63]. In Zucker rats, a single dose of $10 \mathrm{mg} / \mathrm{kg}$ AZD7545 increased muscle PDH activity and chronic treatment improves blood glucose profiles [63]. Notably, this is dependent upon the animal being well fed, as fasted animals dramatically upregulate PDK4 which is not inhibited by AZD7545 and may circumvent this effect.

Although AZD7545 is a potent inhibitor of PDK2, it is surprisingly a stimulator of PDKs in the absence of the E2P/E3BP core [64]. In the case of PDK4, this appears to be due to the locking of PDK4 into a open confirmation when complexed with AZD7545 that enhances its activity. Given this surprising change in activity, it has been proposed that AZD7545 is unlikely to be a successful PDK inhibitor long term as its PDK inhibitory activity is highly dependent on which PDK is being expressed [6]. AZD7545 may be useful for diseases where PDK2 is the predominant enzyme and has a major pathological role as increased PDK4 activity would not necessarily cause any direct harm. PDK and the interactions with notable PDK inhibitors is noted in Figure 2. 


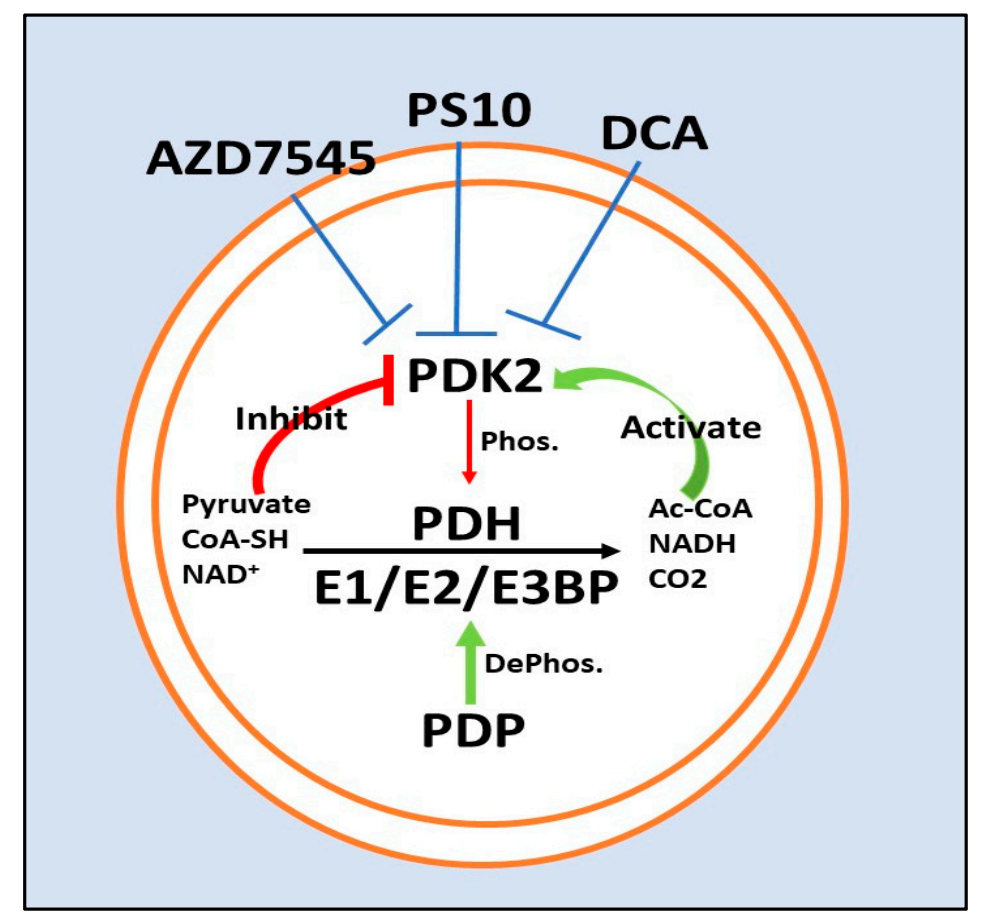

Figure 2. Regulation of PDK2 by allosteric regulators and known inhibitors. Pyruvate is converted to acetyl-CoA by the PDH complex. PDK2 phosphorylates and inhibits pyruvate dehydrogenase in the pyruvate dehydrogenase complex. PDP dephosphorylates PDH and re-activates it. PDK2 is inhibited (red arrow) by pyruvate, $\mathrm{NAD}^{+}$and more. PDK2 is stimulated (green arrow) by acetyl$\mathrm{CoA}, \mathrm{NADH}$ and more. The primary purpose of PDK2 is sustained regulation of PDH to allow for metabolic adaptation to alterations in nutrient conditions. These allosteric regulators keep tight control. Inhibition of PDK2 by DCa, PS10 or AD7545 has been proposed as a means for preventing disease in multiple disorders. $\mathrm{PDH}-$ pyruvate dehydrogenase, CoA-coenzyme A, PDK2-pyruvate dehydrogenase kinase 2, DCA—dichloroacetate, PDP—pyruvate dehydrogenase phosphatase, E1-pyruvate dehydrogenase E1 complex, E2-pyruvate dehydrogenase E2 complex, and $\mathrm{E} 3 / \mathrm{E} 3 \mathrm{BP}$ - pyruvate dehydrogenase E3 complex or E3 binding protein.

\section{Pathophysiological Roles of PDKs in Disease}

Constantly converting pyruvate to acetyl-CoA is highly dependent on stable levels of glucose intake. During many different disease states, and during periods of normal fasting or nutrient insufficiency, glucose becomes less available (fasting/starvation), excessive (dietary overconsumption) or dysregulated intracellularly (cancer, others). During these periods, the body uses other mechanisms to adapt and the PDK isozymes serves as a switch to mediate this change. Metabolic dysregulation is common in these disorders, and targeting PDK has become a common theme for potentially normalizing metabolism and reducing disease phenotype.

\subsection{PDK2 and the Metabolic Syndrome PDK2 and Nutrient Excess}

Non-alcoholic fatty liver disease (NAFLD) and the more progressive form known as non-alcoholic steatohepatitis (NASH) are thought to be the result of excess nutrient accumulation $[65,66]$. This alters metabolism, resulting in accumulation of triglycerides in the liver and leads to insulin resistance, prominent inflammation, and subsequent activation of stellate cells, resulting in liver fibrosis and eventual cirrhosis [65]. NASH/NAFLD are commonly associated with insulin resistance, or type 2 diabetes due to overconsumption of nutrients. As of yet, there are no small-molecule inhibitors that have been approved for the treatment of NAFLD/NASH although clinical trials with the synthetic bile acid obeticholic acid are promising [66]. 
Similar to physiological nutrient deprivation, PDK4 appears to be the primary regulator of the liver's response to nutrient excess. This extends to both metabolic syndrome classically identified by insulin resistance and diabetes, but also nutrient/calorie excess leading hepatic steatosis and eventually steatohepatitis. Although PDK4 has been studied more extensively, PDK2 also appears to potentially play a role in these disease states, especially in concert with PDK4 during loss or inhibition of PDK4. Dual inhibition of PDK2/PDK4 may present a more rationale drug response.

PDK2 deficiency reduces hepatic steatosis and insulin resistance in a model of high fat diet-induced steatosis [25]. While diet-induced steatosis dramatically upregulates PDK4 in skeletal muscle, it downregulates PDK4 in the liver [25]. In contrast, PDK2 levels are upregulated in the liver during diet-induced steatosis [25].This effect was eventually attributed to increased ketogenesis and increased $\beta$-oxidation due to reduced numbers of TCA cycle intermediates (reduced anaplerosis) as increased B-oxidation would break down fat stores and reduce steaosis. In contrast, while similar results were later found after diet-induced obesity was treated therapeutically with PS10, or initiated in double knockout PDK2 $2^{-/} / \mathrm{PDK}^{-/-}$animals, these results were not attributed to reduced anaplerotic input, but rather increased anaplerotic flux [67]. This distinction is important as it indicates that the primary mechanism is increased usage of fatty acids to meet energy demands, and the apparent reduction in TCA intermediates is a result of rapid usage. Recent improvements to PS10 have yielded compounds with preferential uptake in the liver that also combat dietinduced obesity, and thus may avoid off-target toxicity in other tissues [61]. Importantly either PS10 treatment, knockout of the individual PDKs 2 or 4, or knockout of both PDK2 and PDK4 are all protective against hepatic steatosis and insulin insensitivity; although, more severe forms of the disease may not be responsive to this treatment. NAFLD is a largely silent disease that is difficult to identify in patients and does not always progress to NASH. It will be important to determine whether early intervention against the "metabolic phenotype" and reductions in steatosis can also prevent long-term progression to the more severe phenotype. It should be noted that bariatric surgery is an effective treatment for $\mathrm{NASH}$, and thus it stands to reason that management of metabolism may be an effective treatment for reducing NASH progression and improving outcomes [68].

\subsection{PDK2 in Cancer}

Since the initial report that DCA might be an effective cancer therapeutic, PDKs have been widely examined as mediators of cancer biology [69]. Otto Warburg originally proposed in the 1950s that cancer cells preferentially used glycolysis to fuel ATP production $[2,3]$. While modern research indicates that this is only true in part, what is increasingly agreed upon is that alterations in cancer metabolism are broadly aimed at promoting cancer cell proliferation and resistance to apoptosis through metabolic flexibility $[4,5,70]$. PDKs, including PDK2, are primary mediators of Warburg metabolism [5]. Because of extensive regulation of the expression of PDKs, overexpression or suppression of PDKs has been consistently cited as both a means through which some mediators regulate the growth of cancer, and as potential drug targets for inhibiting the growth of cancer $[5,70]$.

Different mechanisms have been proposed for how overexpression of PDKs might enhance the formation of cancer. One of the primary explanations is that inactivation of the pyruvate dehydrogenase complex by the PDKs increases production of ATP by aerobic glycolysis [2,3]. Blocking the oxidation of pyruvate conserves pyruvate for reduction to lactate, therefore regenerating $\mathrm{NAD}^{+}$required for the reaction catalyzed by glyceraldehyde 3-dehydrogenase without a requirement for the malate/aspartate or glycerol phosphate shuttle nor oxygen for the oxidation of cytoplasm NADH. Reducing acetyl-CoA formation also reduces citrate formation and ATP production, minimizing regulation of phosphofructokinase, the rate-limiting enzyme of glycolysis which could enhance ATP production through glycolysis. Mitochondria in cancer are highly active though, and it would be expected that they are highly capable of producing ATP [71]. It has been proposed that the likely advantage provided by aerobic glycolysis is through generation of metabolic 
necessities such as $\mathrm{NAD}^{+}$or other agents through altered means [70]. Thus, another recent explanation is that it is likely that PDK overexpression alters pyruvate metabolism sufficiently such that cancer cells can increase production of TCA intermediates through anaplerotic reactions carried out by pyruvate carboxylase and glutamate dehydrogenase that propel cellular growth [72-74]. In support of these arguments, inhibition of pyruvate carboxylase can dramatically reduce cancer proliferation, and recent data indicate that $\mathrm{NADH} / \mathrm{NAD}$ ratio alterations caused by inhibition of PDKs are partially responsible for their effect $[70,72,73]$. There also remains the possibility that PDKs can phosphorylate other targets than simply PDH; however, this has been only minimally studied thus far [15]. This is an area of intense research interest as a better understanding of the detailed mechanisms associated with PDK expression and cancer is needed to progress both a mechanistic understanding of cancer metabolism and drug development in this area.

\section{PDK2 in Hepatocellular Carcinoma}

Hepatocellular carcinoma (HCC) is a devastating disease with incredibly poor outcomes. Surgical intervention remains the primary form of treatment as chemoresistance is common with HCC, and the only known effective small-molecule inhibitor is sorafenib, which offers only modest increases in lifespan [75]. Interventions against PDKs, particularly PDK2, have proven to be potentially therapeutic in laboratory models of HCC.

Expression of PDKs in HCC remains a topic of interest, although no consensus has been achieved as of yet. Retinoic acid-related orphan receptor $\alpha(\operatorname{ROR} \alpha)$ has been demonstrated to regulate PDKs in HCC cells. Activation of ROR $\alpha$ downregulates PDK2 in HepG2 and Hep3B cells and also reduces phosphorylation of PDH E1 $\alpha$ [33]. Either activation of ROR $\alpha$ or knockdown of PDK2 also slows tumor growth, and importantly, the activation of ROR $\alpha$ appears to be specific to PDK2 as no differences in PDK1, PDK3 or PDK4 were observed [33]. These data indicate that the use of PDK2 inhibitors directly may be useful in HCC, with or without concurrent chemotherapy. The situation may be more complicated though. There is also evidence that PDKs are suppressed in HCC [10,11,76]. Moreover, in orthotopic H4IIE hepatoma bearing rats, PDH activity was found to be higher as PDK2 and PDK4 were both natively downregulated [76]. If PDKs are already downregulated prior to attempts at enzymatic inhibition, it does not seem likely that inhibition of PDKs will be an effective means for blocking tumor growth as they are natively suppressed. In spite of the aforementioned changes, genetic inhibition of PDK2 or pharmacological inhibition of all PDKs with DCA reduces growth of hepatic tumors [77-79]. Moreover, inhibition of PDKs with DCA helps overcome sorafenib resistance and enhances the effect of the common chemotherapeutic agent adriamycin [78,79]. Similarly, DCA was found to enhance the efficacy of oncolytic Newcastle Disease virus and improved its capacity to kill HCC cells in murine models [80]. PDK inhibition seems to improve various liver treatments despite the native downregulation of PDK2 and PDK4. Whether or not PDK2 inhibition could serve as a therapeutic target, with or without co-inhibition of PDK4, remains to be determined. PDK2 inhibition has been proposed as a therapeutic in other cancers and thus this may be tissue and cancer type dependent.

\section{PDKs in Inflammation, a Growing Area of Interest}

Immune populations require massive proliferative capacity in response to infection. The metabolic demands for this proliferative capacity mimic other hyper-proliferative phenotypes such as what is seen with cancer [81]. Not surprisingly, the metabolic demands of this proliferative effort can be limiting if cells cannot find sufficient resources to duplicate. Moreover, activation of T cells and switching between pro-inflammatory (M1) and antiinflammatory (M2) macrophage polarization have recently been linked to PDKs [82,83]. PDK upregulation is linked to M1 polarization checkpoint in macrophages [84]. In contrast to changes observed with blood glucose levels, neither PDK2- nor PDK4-deficient mice have any issue with conversion to M1; however, combined inhibition of PDK2 and PDK4 results in prevention of conversion to M1 phenotype [83]. This is critical as M2 macrophages are 
thought to require higher levels of oxidative phosphorylation than their M1 counterparts which primarily use glycolysis [85]. Recent reports have identified PDKs as direct negative regulators of IL-10, although mechanistically this may not occur through IL-10:PDK interactions, but rather broad metabolic regulation through adenosine monophosphate-activated protein kinase (AMPK) [86]. Inhibition of PDK2/4 should enhance oxidative phosphorylation and reduce glycolysis, consistent with the observed phenotype. Inhibition of PDKs may be an novel means for enhancing immune resolution by preventing the conversion to M1 phenotype in macrophages.

PDK effects in immune cells may be underappreciated relative to their effects on the liver in disease. Importantly, prior observed protection against insulin resistance in PDK2/PDK4 double knockout mice was still observed when PDK2/PDK4 were only knocked out of the hematopoietic cells, indicating that adipose tissue inflammation may be critically involved in this process, possibly due to reduced cytokine secretion from PDK2/PDK4-deficient macrophages [83]. Macrophages are also critical regulators of disease in both alcoholic hepatitis and NAFLD/NASH $[65,66,87]$. Promotion of a proresolution phenotype is likely to benefit both disease states due to note chronic and unresolved inflammation through inhibition of PDKs in cells other than hepatocytes. It should be noted that T cells in which PDK1 is inhibited similarly have issues with release of cytokines, and thus cytokine secretion may be partially dependent on PDKs, likely through some sort of metabolic function [82]. Other studies indicate that administration of DCA or AZD7545 may also functionally prevent Th17 differentiation and push cells towards Treg differentiation. This would likely be deleterious to cancer treatment, as Tregs are an immunosuppressive population that would dampen $\mathrm{T}$ cells responses and normal cancer immunity. This is a fairly novel area of research, and more studies are desperately needed to better understand if PDK inhibition adversely affects some of these other factors.

\section{Conclusions}

Because of the highly inducible nature of PDK4, it is has been the focus of considerable research in the liver field. During this period, PDK2 has been relatively understudied in comparison. In spite of this, PDK2 obviously shares many of the same actions as PDK4 and, given its expression, will likely have to be considered as an additional therapeutic necessity if therapeutic PDK inhibition is to be achieved in the liver. Fortunately, in part due to substantial overlap in their homology, current inhibitors have significant activity at both PDK2 and PDK4. Activation of the PDH complex by inhibition of PDK2, PDK4, or both is likely to be beneficial in numerous disease states, especially if effective, safe inhibitors can be identified. Increased understanding of the dual roles of PDK2 and PDK4 is needed to further refine inhibitors and develop successful preclinical candidates.

Author Contributions: Authors contributed equally to this manuscript. B.L.W. wrote, edited, and conceived the manuscript. R.A.H. wrote, edited and conceived the manuscript. All authors have read and agreed to the published version of the manuscript.

Funding: Research funding for Woolbright's lab was provided by the American Urological Foundation Research Scholar Award.

Conflicts of Interest: The authors declare no conflict of interest.

\section{References}

1. Bowker-Kinley, M.M.; Davis, I.W.; Wu, P.; Harris, A.R.; Popov, M.K. Evidence for existence of tissue-specific regulation of the mammalian pyruvate dehydrogenase complex. Biochem. J. 1998, 329, 191-196. [CrossRef] [PubMed]

2. Warburg, O. On respiratory impairment in cancer cells. Science 1956, 124, 269-270. [PubMed]

3. Warburg, O. On the Origin of Cancer Cells. Science 1956, 123, 309-314. [CrossRef]

4. Stacpoole, P.W. Therapeutic Targeting of the Pyruvate Dehydrogenase Complex/Pyruvate Dehydrogenase Kinase (PDC/PDK) Axis in Cancer. J. Natl. Cancer Inst. 2017, 109. [CrossRef] [PubMed]

5. Woolbright, B.L.; Rajendran, G.; Harris, R.A.; Taylor, J.A. Metabolic Flexibility in Cancer: Targeting the Pyruvate Dehydrogenase Kinase:Pyruvate Dehydrogenase Axis. Mol. Cancer Ther. 2019, 18, 1673-1681. [CrossRef] 
6. Klyuyeva, A.; Tuganova, A.; Kedishvili, N.Y.; Popov, K.M. Tissue-specific kinase expression and activity regulate flux through the pyruvate dehydrogenase complex. J. Biol. Chem. 2019, 294, 838-851. [CrossRef]

7. Huang, B.; Wu, P.; Bowker-Kinley, M.M.; Harris, R.A. Regulation of Pyruvate Dehydrogenase Kinase Expression by Peroxisome Proliferator-Activated Receptor- Ligands, Glucocorticoids, and Insulin. Diabetes 2002, 51, 276-283. [CrossRef]

8. Sugden, M.C.; Holness, M.J. Mechanisms underlying regulation of the expression and activities of the mammalian pyruvate de-hydrogenase kinases. Arch Physiol. Biochem. 2006, 112, 139-149. [CrossRef]

9. Jeoung, N.H.; Harris, R.A. Role of Pyruvate Dehydrogenase Kinase 4 in Regulation of Blood Glucose Levels. Korean Diabetes J. 2010, 34, 274-283. [CrossRef]

10. Choiniere, J.; Wu, J.; Wang, L. Pyruvate Dehydrogenase Kinase 4 Deficiency Results in Expedited Cellular Proliferation through E2F1-Mediated Increase of Cyclins. Mol. Pharmacol. 2016, 91, 189-196. [CrossRef]

11. Choiniere, J.; Lin, M.J.; Wang, L.; Wu, J. Deficiency of pyruvate dehydrogenase kinase 4 sensitizes mouse liver to diethylnitrosamine and arsenic toxicity through inducing apoptosis. Liver Res. 2018, 2, 100-107. [CrossRef]

12. Jeoung, N.H.; Rahimi, Y.; Wu, P.; Lee, W.N.P.; Harris, R.A. Fasting induces ketoacidosis and hypothermia in PDHK2/PDHK4double-knockout mice. Biochem. J. 2012, 443, 829-839. [CrossRef]

13. Linn, T.C.; Pettit, F.H.; Reed, L.J. Alpha-keto acid dehydrogenase complexes. X. Regulation of the activity of the pyruvate dehy-drogenase complex from beef kidney mitochondria by phosphorylation and dephosphorylation. Proc. Natl. Acad. Sci. USA 1969, 62, 234-241. [CrossRef]

14. Roche, T.E.; Baker, J.C.; Yan, X.; Hiromasa, Y.; Gong, X.; Peng, T.; Dong, J.; Turkan, A.; Kasten, S.A. Distinct regulatory properties of pyruvate dehydrogenase kinase and phosphatase isoforms. Prog. Nucleic Acid Res. Mol. Biol. 2001, 70, 33-75. [CrossRef] [PubMed]

15. Shi, G.; McQuibban, G.A. The Mitochondrial Rhomboid Protease PARL Is Regulated by PDK2 to Integrate Mitochondrial Quality Control and Metabolism. Cell Rep. 2017, 18, 1458-1472. [CrossRef] [PubMed]

16. Jeon, J.; Thoudam, T.; Choi, E.J.; Kim, M.; Harris, R.A.; Lee, I. Loss of metabolic flexibility as a result of overexpression of pyruvate dehydrogenase kinases in muscle, liver and the immune system: Therapeutic targets in metabolic diseases. J. Diabetes Investig. 2021, 12, 21-31. [CrossRef] [PubMed]

17. Kato, M.; Li, J.; Chuang, J.L.; Chuang, D.T. Distinct Structural Mechanisms for Inhibition of Pyruvate Dehydrogenase Kinase Isoforms by AZD7545, Dichloroacetate, and Radicicol. Structure 2007, 15, 992-1004. [CrossRef] [PubMed]

18. Klyuyeva, A.; Tuganova, A.; Popov, K.M. Allosteric Coupling in Pyruvate Dehydrogenase Kinase 2. Biochemistry 2008, 47, 8358-8366. [CrossRef] [PubMed]

19. Sugden, M.C.; Holness, M.J. Recent advances in mechanisms regulating glucose oxidation at the level of the pyruvate dehydrogenase complex by PDKs. Am. J. Physiol. Endocrinol. Metab. 2003, 284, E855-E862. [CrossRef] [PubMed]

20. Kim, J.-W.; Tchernyshyov, I.; Semenza, G.L.; Dang, C.V. HIF-1-mediated expression of pyruvate dehydrogenase kinase: A metabolic switch required for cellular adaptation to hypoxia. Cell Metab. 2006, 3, 177-185. [CrossRef] [PubMed]

21. Sun, W.; Zhou, S.; Chang, S.S.; McFate, T.; Verma, A.; Califano, J.A. Mitochondrial Mutations Contribute to HIF1 Accumulation via Increased Reactive Oxygen Species and Up-regulated Pyruvate Dehydrogenease Kinase 2 in Head and Neck Squamous Cell Carcinoma. Clin. Cancer Res. 2009, 15, 476-484. [CrossRef]

22. Dunford, E.C.; Herbst, E.A.; Jeoung, N.H.; Gittings, W.; Inglis, J.G.; Vandenboom, R.; Leblanc, P.J.; Harris, R.A.; Peters, S.J. PDH activation during in vitro muscle contractions in PDH kinase 2 knockout mice: Effect of PDH kinase 1 compensation. Am. J. Physiol. Integr. Comp. Physiol. 2011, 300, R1487-R1493. [CrossRef]

23. Fuller, S.J.; Randle, P.J. Reversible phosphorylation of pyruvate dehydrogenase in rat skeletal-muscle mitochondria. Effects of starvation and diabetes. Biochem. J. 1984, 219, 635-646. [CrossRef]

24. Jeong, J.Y.; Jeoung, N.H.; Park, K.-G.; Lee, I.-K. Transcriptional Regulation of Pyruvate Dehydrogenase Kinase. Diabetes Metab. J. 2012, 36, 328-335. [CrossRef] [PubMed]

25. Go, Y.; Jeong, J.Y.; Jeoung, N.H.; Jeon, J.-H.; Park, B.-Y.; Kang, H.-J.; Ha, C.-M.; Choi, Y.-K.; Lee, S.J.; Ham, H.J.; et al. Inhibition of Pyruvate Dehydrogenase Kinase 2 Protects Against Hepatic Steatosis Through Modulation of Tricarboxylic Acid Cycle Anaplerosis and Ketogenesis. Diabetes 2016, 65, 2876-2887. [CrossRef]

26. Wu, P.; Sato, J.; Zhao, Y.; Jaskiewicz, J.; Popov, M.K.; Harris, A.R. Starvation and diabetes increase the amount of pyruvate dehydrogenase kinase isoenzyme 4 in rat heart. Biochem. J. 1998, 329, 197-201. [CrossRef]

27. Majer, M.; Popov, K.M.; Harris, R.A.; Bogardus, C.; Prochazka, M. Insulin Downregulates Pyruvate Dehydrogenase Kinase (PDK) mRNA: Potential Mechanism Contributing to Increased Lipid Oxidation in Insulin-Resistant Subjects. Mol. Genet. Metab. 1998, 65, 181-186. [CrossRef] [PubMed]

28. Kwon, H.-S.; Huang, B.; Unterman, T.G.; Harris, R.A. Protein Kinase B- Inhibits Human Pyruvate Dehydrogenase Kinase-4 Gene Induction by Dexamethasone Through Inactivation of FOXO Transcription Factors. Diabetes 2004, 53, 899-910. [CrossRef] [PubMed]

29. Furuyama, T.; Kitayama, K.; Yamashita, H.; Mori, N. Forkhead transcription factor FOXO1 (FKHR)-dependent induction of PDK4 gene expression in skeletal muscle during energy deprivation. Biochem. J. 2003, 375, 365-371. [CrossRef] [PubMed]

30. Rena, G.; Guo, S.; Cichy, S.C.; Unterman, T.G.; Cohen, P. Phosphorylation of the Transcription Factor Forkhead Family Member FKHR by Protein Kinase B. J. Biol. Chem. 1999, 274, 17179-17183. [CrossRef] [PubMed] 
31. Zhang, W.; Patil, S.; Chauhan, B.; Guo, S.; Powell, D.R.; Le, J.; Klotsas, A.; Matika, R.; Xiao, X.; Franks, R.; et al. FoxO1 regulates multiple metabolic pathways in the liver: Effects on gluconeogenic, glycolytic, and lipogenic gene expression. J. Biol. Chem. 2006, 281, 10105-10117. [CrossRef] [PubMed]

32. Degenhardt, T.; Saramäki, A.; Malinen, M.; Rieck, M.; Väisänen, S.; Huotari, A.; Herzig, K.H.; Müller, R.; Carlberg, C. Three members of the human pyruvate dehydrogenase kinase gene family are direct targets of the peroxisome proliferator-activated receptor beta/delta. J. Mol. Biol. 2007, 372, 341-355. [CrossRef] [PubMed]

33. Byun, J.; Choi, Y.; Na Kang, Y.; Jang, B.K.; Kang, K.J.; Jeon, Y.H.; Lee, H.; Jeon, J.; Koo, S.; Jeong, W.; et al. Retinoic acid-related orphan receptor alpha reprograms glucose metabolism in glutamine-deficient hepatoma cells. Hepatology 2015, 61, 953-964. [CrossRef] [PubMed]

34. Contractor, T.; Harris, C.R. p53 Negatively Regulates Transcription of the Pyruvate Dehydrogenase Kinase Pdk2. Cancer Res. 2012, 72, 560-567. [CrossRef] [PubMed]

35. Roche, T.E.; Hiromasa, Y. Pyruvate dehydrogenase kinase regulatory mechanisms and inhibition in treating diabetes, heart ischemia, and cancer. Cell. Mol. Life Sci. 2007, 64, 830-849. [CrossRef] [PubMed]

36. Steussy, C.N.; Popov, K.M.; Bowker-Kinley, M.M.; Sloan, R.B., Jr.; Harris, R.A.; Hamilton, J.A. Structure of pyruvate dehydrogenase kinase. Novel folding pattern for a serine protein kinase. J. Biol. Chem. 2001, 276, 37443-37450. [CrossRef]

37. Brough, P.A.; Aherne, W.; Barril, X.; Borgognoni, J.; Boxall, K.; Cansfield, J.E.; Cheung, K.-M.J.; Collins, I.; Davies, N.G.M.; Drysdale, M.J.; et al. 4,5-Diarylisoxazole Hsp90 Chaperone Inhibitors: Potential Therapeutic Agents for the Treatment of Cancer. J. Med. Chem. 2008, 51, 196-218. [CrossRef] [PubMed]

38. Moore, J.D.; Staniszewska, A.; Shaw, T.; D'Alessandro, J.; Davis, B.; Surgenor, A.; Baker, L.; Matassova, N.; Murray, J.; Macias, A.; et al. VER-246608, a novel pan-isoform ATP competitive inhibitor of pyruvate dehydrogenase kinase, disrupts Warburg metabolism and induces context-dependent cytostasis in cancer cells. Oncotarget 2014, 5, 12862-12876. [CrossRef]

39. Tuganova, A.; Yoder, M.D.; Popov, K.M. An Essential Role of Glu-243 and His-239 in the Phosphotransfer Reaction Catalyzed by Pyruvate Dehydrogenase Kinase. J. Biol. Chem. 2001, 276, 17994-17999. [CrossRef]

40. Sharma, S.V.; Agatsuma, T.; Nakano, H. Targeting of the protein chaperone, HSP90, by the transformation suppressing agent, radicicol. Oncogene 1998, 16, 2639-2645. [CrossRef]

41. Tso, S.-C.; Qi, X.; Gui, W.-J.; Wu, C.-Y.; Chuang, J.L.; Wernstedt-Asterholm, I.; Morlock, L.K.; Owens, K.R.; Scherer, P.E.; Williams, N.S.; et al. Structure-guided Development of Specific Pyruvate Dehydrogenase Kinase Inhibitors Targeting the ATP-binding Pocket. J. Biol. Chem. 2014, 289, 4432-4443. [CrossRef] [PubMed]

42. Pratt, M.L.; Roche, T.E. Mechanism of pyruvate inhibition of kidney pyruvate dehydrogenasea kinase and synergistic inhibition by pyruvate and ADP. J. Biol. Chem. 1979, 254, 7191-7196. [CrossRef]

43. Knoechel, T.R.; Tucker, A.D.; Robinson, C.M.; Phillips, C.; Taylor, W.; Bungay, P.J.; Kasten, S.A.; Roche, T.E.; Brown, D.G. Regulatory roles of the N-terminal domain based on crystal structures of human pyruvate dehydrogenase kinase 2 containing physiological and synthetic ligands. Biochemistry 2006, 45, 402-415. [CrossRef]

44. Holness, M.; Sugden, M. Regulation of pyruvate dehydrogenase complex activity by reversible phosphorylation. Biochem. Soc. Trans. 2003, 31, 1143-1151. [CrossRef] [PubMed]

45. Li, J.; Kato, M.; Chuang, D.T. Pivotal Role of the C-terminal DW-motif in Mediating Inhibition of Pyruvate Dehydrogenase Kinase 2 by Dichloroacetate. J. Biol. Chem. 2009, 284, 34458-34467. [CrossRef] [PubMed]

46. Whitehouse, S.; Cooper, R.H.; Randle, P.J. Mechanism of activation of pyruvate dehydrogenase by dichloroacetate and other halogenated carboxylic acids. Biochem. J. 1974, 141, 761-774. [CrossRef]

47. Babu, E.; Ramachandran, S.; Coothankandaswamy, V.; Elangovan, S.; Prasad, P.D.; Ganapathy, V.; Thangaraju, M. Role of SLC5A8, a plasma membrane transporter and a tumor suppressor, in the antitumor activity of dichloroacetate. Oncogene 2011, 30, 4026-4037. [CrossRef]

48. James, M.O.; Jahn, S.C.; Zhong, G.; Smeltz, M.G.; Hu, Z.; Stacpoole, P.W. Therapeutic applications of dichloroacetate and the role of glutathione transferase zeta-1. Pharmacol. Ther. 2017, 170, 166-180. [CrossRef]

49. Jahn, S.C.; Solayman, M.H.M.; Lorenzo, R.J.; Langaee, T.; Stacpoole, P.W.; James, M.O. GSTZ1 expression and chloride concentrations modulate sensitivity of cancer cells to dichloroacetate. Biochim. Biophys. Acta Gen. Subj. 2016, 1860, 1202-1210. [CrossRef]

50. Stacpoole, P.W. The pharmacology of dichloroacetate. Metabolism 1989, 38, 1124-1144. [CrossRef]

51. Stacpoole, P.W.; Nagaraja, N.V.; Hutson, A.D. Efficacy of dichloroacetate as a lactate-lowering drug. J. Clin. Pharmacol. 2003, 43, 683-691. [CrossRef] [PubMed]

52. Shroads, A.L.; Langaee, T.; Coats, B.S.; Kurtz, T.L.; Bullock, J.R.; Weithorn, D.; Gong, Y.; Wagner, D.A.; Ostrov, D.A.; Johnson, J.A.; et al. Human Polymorphisms in the Glutathione Transferase Zeta 1/Maleylacetoacetate Isomerase Gene Influence the Toxicokinetics of Dichloroacetate. J. Clin. Pharmacol. 2012, 52, 837-849. [CrossRef] [PubMed]

53. Dunbar, E.M.; Coats, B.S.; Shroads, A.L.; Langaee, T.; Lew, A.; Forder, J.R.; Shuster, J.J.; Wagner, D.A.; Stacpoole, P.W. Phase 1 trial of dichloroacetate (DCA) in adults with recurrent malignant brain tumors. Investig. New Drugs 2014, 32, 452-464. [CrossRef] [PubMed]

54. Stacpoole, P.W.; Martyniuk, C.J.; James, M.O.; Calcutt, N.A. Dichloroacetate-induced peripheral neuropathy. Int. Rev. Neurobiol. 2019, 145, 211-238. [CrossRef] 
55. Tataranni, T.; Agriesti, F.; Pacelli, C.; Ruggieri, V.; Laurenzana, I.; Mazzoccoli, C.; Della-Sala, G.; Panebianco, C.; Pazienza, V.; Capitanio, N.; et al. Dichloroacetate Affects Mitochondrial Function and Stemness-Associated Properties in Pancreatic Cancer Cell Lines. Cells 2019, 8, 478. [CrossRef] [PubMed]

56. Tataranni, T.; Piccoli, C. Dichloroacetate (DCA) and Cancer: An Overview towards Clinical Applications. Oxidative Med. Cell. Longev. 2019, 2019, 1-14. [CrossRef]

57. Chen, J.; Guccini, I.; di Mitri, D.; Brina, D.; Revandkar, A.; Sarti, M.; Pasquini, E.; Alajati, A.; Pinton, S.; Losa, M.; et al. Compartmentalized activities of the pyruvate dehydrogenase complex sustain lipogenesis in prostate cancer. Nat. Genet. 2018, 50, 219-228. [CrossRef]

58. Zachar, Z.; Marecek, J.; Maturo, C.; Gupta, S.; Stuart, S.D.; Howell, K.; Schauble, A.; Lem, J.; Piramzadian, A.; Karnik, S.; et al. Non-redox-active lipoate derivates disrupt cancer cell mitochondrial metabolism and are potent anticancer agents in vivo. J. Mol. Med. 2011, 89, 1137-1148. [CrossRef]

59. Newsholme, E.A.; Crabtree, B.; Ardawi, M.S.M. The role of high rates of glycolysis and glutamine utilization in rapidly dividing cells. Biosci. Rep. 1985, 5, 393-400. [CrossRef]

60. Dutta, R.; Inouye, M. GHKL, an emergent ATPase/kinase superfamily. Trends Biochem. Sci. 2000, 25, 24-28. [CrossRef]

61. Tso, S.-C.; Lou, M.; Wu, C.-Y.; Gui, W.-J.; Chuang, J.L.; Morlock, L.K.; Williams, N.S.; Wynn, R.M.; Qi, X.; Chuang, D.T. Development of Dihydroxyphenyl Sulfonylisoindoline Derivatives as Liver-Targeting Pyruvate Dehydrogenase Kinase Inhibitors. J. Med. Chem. 2017, 60, 1142-1150. [CrossRef] [PubMed]

62. Morrell, J.; Orme, J.; Butlin, R.; Roche, T.; Mayers, R.; Kilgour, E. AZD7545 is a selective inhibitor of pyruvate dehydrogenase kinase 2. Biochem. Soc. Trans. 2003, 31, 1168-1170. [CrossRef] [PubMed]

63. Mayers, R.; Butlin, R.; Kilgour, E.; Leighton, B.; Martin, D.; Myatt, J.; Orme, J.; Holloway, B. AZD7545, a novel inhibitor of pyruvate dehydrogenase kinase 2 (PDHK2), activates pyruvate dehydrogenase in vivo and improves blood glucose control in obese (fa/fa) Zucker rats. Biochem. Soc. Trans. 2003, 31, 1165-1167. [CrossRef] [PubMed]

64. Wynn, R.M.; Kato, M.; Chuang, J.L.; Tso, S.-C.; Li, J.; Chuang, D.T. Pyruvate Dehydrogenase Kinase-4 Structures Reveal a Metastable Open Conformation Fostering Robust Core-free Basal Activity. J. Biol. Chem. 2008, 283, 25305-25315. [CrossRef] [PubMed]

65. Arrese, M.; Cabrera, D.; Kalergis, A.M.; Feldstein, A.E. Innate Immunity and Inflammation in NAFLD/NASH. Dig. Dis. Sci. 2016, 61, 1294-1303. [CrossRef] [PubMed]

66. Rowe, I.A.; Wong, V.W.-S.; Loomba, R. Treatment candidacy for pharmacologic therapies for NASH. Clin. Gastroenterol. Hepatol. 2021. [CrossRef] [PubMed]

67. Wu, C.Y.; Tso, S.C.; Chuang, J.L.; Gui, W.J.; Lou, M.; Sharma, G.; Khemtong, C.; Qi, X.; Wynn, R.M.; Chuang, D.T. Targeting hepatic pyruvate dehydrogenase kinases restores insulin signaling and mitigates ChREBP-mediated lipo-genesis in diet-induced obese mice. Mol. Metab. 2018, 12, 12-24. [CrossRef]

68. Lassailly, G.; Caiazzo, R.; Ntandja-Wandji, L.-C.; Gnemmi, V.; Baud, G.; Verkindt, H.; Ningarhari, M.; Louvet, A.; Leteurtre, E.; Raverdy, V.; et al. Bariatric Surgery Provides Long-term Resolution of Nonalcoholic Steatohepatitis and Regression of Fibrosis. Gastroenterology 2020, 159, 1290-1301. [CrossRef] [PubMed]

69. Bonnet, S.; Archer, S.L.; Allalunis-Turner, J.; Haromy, A.; Beaulieu, C.; Thompson, R.; Lee, C.T.; Lopaschuk, G.D.; Puttagunta, L.; Bonnet, S.; et al. A mitochondria-K+ channel axis is suppressed in cancer and its normalization promotes apoptosis and inhibits cancer growth. Cancer Cell 2007, 11,37-51. [CrossRef] [PubMed]

70. Heiden, M.G.V.; Cantley, L.C.; Thompson, C.B. Understanding the Warburg effect: The metabolic requirements of cell proliferation. Science 2009, 324, 1029-1033. [CrossRef]

71. Wallace, D.C. Mitochondria and cancer. Nat. Rev. Cancer 2012, 12, 685-698. [CrossRef] [PubMed]

72. Shang, H.; Zheng, J.; Tong, J. Integrated analysis of transcriptomic and metabolomic data demonstrates the significant role of pyruvate carboxylase in the progression of ovarian cancer. Aging 2020, 12, 21874-21889. [CrossRef]

73. Lin, Q.; He, Y.; Wang, X.; Zhang, Y.; Hu, M.; Guo, W.; He, Y.; Zhang, T.; Lai, L.; Sun, Z.; et al. Targeting Pyruvate Carboxylase by a Small Molecule Suppresses Breast Cancer Progression. Adv. Sci. 2020, 7, 1903483. [CrossRef] [PubMed]

74. Moreno-Sánchez, R.; Marín-Hernández, Á.; Gallardo-Pérez, J.C.; Pacheco-Velázquez, S.C.; Robledo-Cadena, D.X.; Padilla-Flores, J.A.; Saavedra, E.; Rodríguez-Enríquez, S. Physiological Role of Glutamate Dehydrogenase in Cancer Cells. Front. Oncol. 2020, 10, 429. [CrossRef] [PubMed]

75. Llovet, J.M.; Ricci, S.; Mazzaferro, V.; Hilgard, P.; Gane, E.; Blanc, J.F.; de Oliveira, A.C.; Santoro, A.; Raoul, J.L.; Forner, A.; et al. Sorafenib in Advanced Hepatocellular Carcinoma. N. Engl. J. Med. 2008, 359, 378-390. [CrossRef] [PubMed]

76. Lee, M.H.; de Berardinis, R.J.; Wen, X.; Corbin, I.R.; Sherry, A.D.; Malloy, C.R.; Jin, E.S. Active pyruvate dehydrogenase and impaired gluconeogenesis in orthotopic hepatomas of rats. Metabolism 2019, 101, 153993. [CrossRef]

77. Fekir, K.; Dubois-Pot-Schneider, H.; Désert, R.; Daniel, Y.; Glaise, D.; Rauch, C.; Morel, F.; Fromenty, B.; Musso, O.; Cabillic, F.; et al. Retrodifferentiation of Human Tumor Hepatocytes to Stem Cells Leads to Metabolic Reprogramming and Chemo-resistance. Cancer Res. 2019, 79, 1869-1883. [CrossRef]

78. Dai, Y.; Xiong, X.; Huang, G.; Liu, J.; Sheng, S.; Wang, H.; Qin, W. Dichloroacetate Enhances Adriamycin-Induced Hepatoma Cell Toxicity In Vitro and In Vivo by Increasing Reactive Oxygen Species Levels. PLoS ONE 2014, 9, e92962. [CrossRef] 
79. Shen, Y.-C.; Ou, D.-L.; Hsu, C.; Lin, K.-L.; Chang, C.-Y.; Lin, C.-Y.; Liu, S.-H.; Cheng, A.-L. Activating oxidative phosphorylation by a pyruvate dehydrogenase kinase inhibitor overcomes sorafenib resistance of hepatocellular carcinoma. Br. J. Cancer 2012, 108, 72-81. [CrossRef]

80. Meng, G.; Li, B.; Chen, A.; Zheng, M.; Xu, T.; Zhang, H.; Dong, J.; Wu, J.; Yu, D.; Wei, J. Targeting aerobic glycolysis by dichloroacetate improves Newcastle disease virus-mediated viro-immunotherapy in hepatocellular carcinoma. Br. J. Cancer 2020, 122, 111-120. [CrossRef]

81. Palmer, C.S.; Ostrowski, M.; Balderson, B.; Christian, N.; Crowe, S.M. Glucose Metabolism Regulates T Cell Activation, Differentiation, and Functions. Front. Immunol. 2015, 6, 1. [CrossRef] [PubMed]

82. Menk, A.V.; Scharping, N.E.; Moreci, R.S.; Zeng, X.; Guy, C.; Salvatore, S.; Bae, H.; Xie, J.; Young, H.A.; Wendell, S.G.; et al. Early TCR Signaling Induces Rapid Aerobic Glycolysis Enabling Distinct Acute T Cell Effector Functions. Cell Rep. 2018, 22, 1509-1521. [CrossRef] [PubMed]

83. Min, B.-K.; Park, S.; Kang, H.-J.; Kim, D.W.; Ham, H.J.; Ha, C.-M.; Choi, B.-J.; Lee, J.Y.; Oh, C.J.; Yoo, E.K.; et al. Pyruvate Dehydrogenase Kinase Is a Metabolic Checkpoint for Polarization of Macrophages to the M1 Phenotype. Front. Immunol. 2019, 10, 944. [CrossRef] [PubMed]

84. Tan, Z.; Xie, N.; Cui, H.; Moellering, D.R.; Abraham, E.; Thannickal, V.J.; Liu, G. Pyruvate Dehydrogenase Kinase 1 Participates in Macrophage Polarization via Regulating Glucose Metabolism. J. Immunol. 2015, 194, 6082-6089. [CrossRef]

85. Galvan-Pena, S.; O’Neill, L.A. Metabolic reprograming in macrophage polarization. Front. Immunol. $2014,5,420$.

86. Na, Y.R.; Jung, D.; Song, J.; Park, J.-W.; Hong, J.J.; Seok, S.H. Pyruvate dehydrogenase kinase is a negative regulator of interleukin10 production in macrophages. J. Mol. Cell Biol. 2020, 12, 543-555. [CrossRef]

87. Woolbright, B.L.; Jaeschke, H. Alcoholic Hepatitis: Lost in Translation. J. Clin. Transl. Hepatol. 2017, 6, 1-8. [CrossRef] 